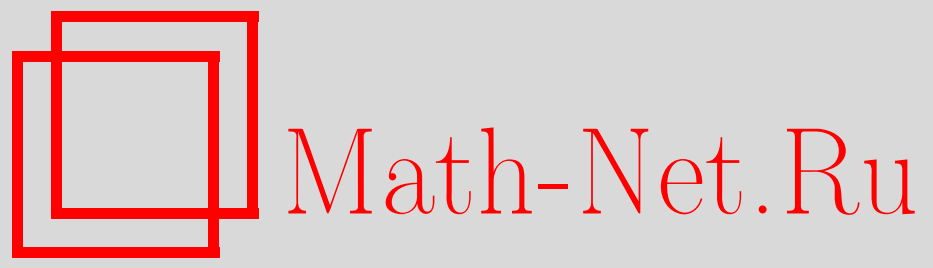

Обцероссийский математический портал

С. Ю. Немировский, Штейновы области с Леви-плоскими границами на компактных комплексных поверхностях, $\mathrm{Ma-}$ тем. заметки, 1999, том 66, выпуск 4, 632-635

DOI: https://doi.org/10.4213/mzm1206

Использование Общероссийского математического портала Math-Net.Ru подразумевает, что вы прочитали и согласны с пользовательским соглашением http://www.mathnet.ru/rus/agreement

Параметры загрузки:

IP : 18.234 .156 .22

26 апреля 2023 г., 17:41:06 


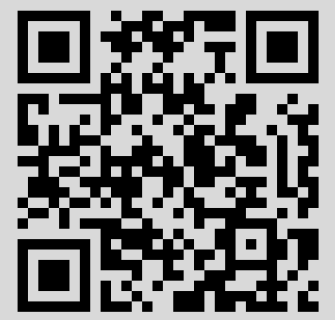




\section{ШТЕЙНОВЫ ОБЛАСТИ С ЛЕВИ-ПЛОСКИМИ ГРАНИЦАМИ НА КОМПАКТНЫХ КОМПЛЕКСНЫХ ПОВЕРХНОСТЯХ}

\section{С. Ю. Немировский}

Введение. Рассмотрим компактную комплексную алгебраическую поверхность $V$. Если $C \subset V$ - гиперплоское сечение, то многообразие $V \backslash C$, очевидно, допускает вложение в $\mathbb{C}^{n}$, т.е. является многообразием Штейна. Взяв в $V \backslash C$ относительно компактную штейнову область с гладкой границей, мы получим разрезание поверхности $V$ на две части, одна из которых штейнова и псевдовыпукла, а другая представляет собой псевдовогнутую окрестность комплексной кривой.

Имеется также другая геометрическая конструкция штейновых областей в $V$. Рассмотрим на поверхности $V$ вложенную вещественную поверхность $S$, гомологичную неособому гиперплоскому сечению $C$ и имеющую род $g(S)=g(C)+\max \left\{0, c_{1}(V) \cdot C\right\}$. При этих условиях поверхность $S$ можно пошевелить $\left(C^{0}\right.$-малой изотопией) так, что у нее появится фундаментальная система штейновых строго псевдовьпуклых окрестностей $U_{\varepsilon} \supset S$ (см., например, [1]). Снова получается разрезание поверхности $V$ на штейнову и псевдовогнутую части, но теперь ни одна из них не содержит компактных комплексных кривых. (Области $U_{\varepsilon}$ пересекают все такие кривые, поскольку для любой комплексной кривой $L \subset V$ индекс пересечения $L \cdot S=L \cdot C>0$.)

Возникает вопрос: можно ли сделать такое разрезание вдоль гладкой вещественной гиперповерхности полностью "симметричным", т.е. добиться, чтобы обе получившиеся области были штейновьми?

Ясно, что в этом случае граница должна быть Леви-плоской (поскольку она псевдовыпукла с обеих сторон). Однако для классических примеров Леви-плоских гиперповерхностей на комплексных поверхностях ограничиваемые ими области как раз не яөляются штейновыми (см. [2]). Заметим, впрочем, что если отказаться от требования гладкости гиперповерхности, то пример появляется тут же: замькание стандартного $\mathbb{R}^{3} \subset \mathbb{C}^{2}$ в $\mathbb{C P}^{2}$ делит проективную плоскость на две штейновы области.

В настоящей заметке мы указываем конструкцию компактных комплексньх поверхностей, допускающих разрезание на две (изоморфные) штейновы части вдоль гладкой Леви-плоской гиперповерхности. Среди этих поверхностей есть как алгебраические (например, прямое произведение любой компактной римановой поверхности на некоторый тор), так и неалгебраические (поверхность Хопфа).

Работа организована следующим образом. Основная конструкция разрезаемых поверхностей и ее многомерный вариант приведены в п. 1. Затем в п. 2 мы рассматриваем частный случай поверхности Хопфа, а в п. 3 изучаем свойства определяющих функций для получившихся штейновых областей с Леви-плоской границей.

Работа выполнена при частичной поддержке Международного научного фонда, грант а98-1785.

(C) С.ю. Немировский 1999 
1. Разрезание эллиптических поверхностей. Наша конструкция основана на следующем классическом наблюдении. Для вещественного $r>1$ рассмотрим факторпространство проколотой комплексной плоскости $\mathbb{C}^{*}=\mathbb{C} \backslash\{0\}$ по отношению эквивалентности $z \sim r z$. Это некоторый комплексный одномерный тор $T$. Образ любой вещественной прямой $l \subset \mathbb{C}$, проходящей через 0 , делит тор $T$ на две половинки, биголоморфно эквивалентные некоторому кольцу $A$.

Теперь осуществим это построение в "относительной ситуации". Пусть $L \rightarrow \Sigma$ - голоморфное линейное расслоение на компактной римановой поверхности. Пусть $s: \Sigma \rightarrow L$-мероморфное сечение расслоения $L$, имеющее простые нули в точках $N_{1}, \ldots, N_{k} \in \Sigma$ и простые полюсы в точках $P_{1}, \ldots, P_{m} \in \Sigma$. Мы предполагаем, что $k+m>0$.

Обозначим $L^{*}=L \backslash\{$ нулевое сечение $\}$ и рассмотрим в $L^{*}$ замыкание множества

$$
\mathbb{R} s:=\left\{\lambda \cdot s(z) \mid z \in \Sigma \backslash\left\{N_{1}, \ldots, N_{k}, P_{1}, \ldots, P_{m}\right\}, \lambda \in \mathbb{R}\right\} .
$$

Лемма. Подмножество $M=\overline{\mathbb{R} s} \subset L^{*}$ есть гладкая Леви-плоская аиперповерхность.

ДоКАЗАТЕЛЬСТвО. Утверждение достаточно проверить только в окрестности нулей и полюсов сечения $s$. Выберем в окрестности такой точки координаты $(z, w)$ в $L$ таким образом, что $s(z)=(z, z)$ или $s(z)=(z, 1 / z)$, если $z=0$ - это нуль или полюс $s$ соответственно. Тогда множество $M$ задается соотношением $\operatorname{Im} w \bar{z}=0$ или $\operatorname{Im} w z=0$. Оба уравнения определяют Леви-плоские гиперповерхности, гладкие вне начала координат в $\mathbb{C}^{2}$.

Заметим, что вещественная гиперповерхность $M$ содержит слои расслоения $L^{*}$ над точками $N_{1}, \ldots, N_{k}, P_{1}, \ldots, P_{m}$ и пересекает все остальные слои по вещественньм прямым.

Рассмотрим теперь на $L^{*}$ отношение эквивалентности $Z \sim r Z$. Факторпространство $X=\pi\left(L^{*}\right)$ представляет собой эллиптическую поверхность, а точнее говоря, локально тривиальное расслоение с базой $\Sigma$ и слоем $T$. Поскольку гиперповерхность $M$, очевидно, замкнута относительно введенной эквивалентности, ее образ $Y=\pi(M)$ является гладкой Леви-плоской гиперповерхностью в $X$ и делит компактную поверхность $X$ на две области $X_{ \pm}$, каждая из которых расслоена над проколотой римановой поверхностью $\Sigma^{\prime}=\Sigma \backslash\left\{N_{1}, \ldots, N_{k}, P_{1}, \ldots, P_{m}\right\}$ со слоем $A$. В действительности, это расслоение является прямым произведением, потому что ограничение $L \rightarrow \Sigma^{\prime}$ тривиально (имеет голоморфное сечение $s$ без нулей). Таким образом, доказано следующее основное

ПрЕДЛОЖЕНИЕ 1. Леви-плоская әиперповерхность $Y$ делит компактную комплексную поверхность $X$ на две штейновы области $X_{ \pm}$, биголоморфно эквивалентные прямому произведению $\Sigma^{\prime} \times A$ проколотой римановой поверхности на кольчо.

ЗАмечАниЕ. В случае, когда $\Sigma=\mathbb{C P}^{1}$, а $s$ - сечение тривиального расслоения с одним нулем и одним полюсом, мы получаем, по существу, область в $X=\mathbb{C P}^{1} \times T$, построенную Осавой [3]. Наша конструкция в каком-то смысле объединяет его пример со случаем поверхности Хопфа, на котором мы подробнее остановимся в следующем пункте.

Приведенное выше построение можно аналогичным образом осуществить в многомерной ситуации. Пусть $V$-проективное многообразие и $L \rightarrow V$-очень обильное линейное расслоение. Тогда для общего сечения $s: V \rightarrow L$ его дивизор $D=\{s=0\}$ является гладкой комплексной гиперповерхностью, а многообразие $V \backslash D$ - аффинным и потому штейновым многообразием.

Повторяя доказательство предложения 1 , мы получаем, что вещественная гиперповерхность $\overline{\mathbb{R} s} \subset L^{*}$ определяет разрезание факторпространства $L^{*} /(Z \sim r Z)$ на две штейновы области, биголоморфно экивалентные прямому произведению $V \backslash D$ на кольцо $A$.

2. Разрезание поверхности Хопфа. Поверхность Хопфа получается из конструкции п. 1 , если $\Sigma=\mathbb{C P}^{1}$ и $s$ - сечение тавтологического линейного расслоения с единственным полюсом. Удобно взглянуть на эту ситуацию "с другой стороны".

Представим поверхность Хопфа как $X=\mathbb{C}^{2} \backslash\{0\} /(Z \sim r Z)$. Тогда $\pi: \mathbb{C}^{2} \backslash\{0\} \rightarrow X-$ естественная проекция, а $M=\mathbb{R}^{3} \subset \mathbb{C}^{2}$ - это проходящая через начало координат вещественная гиперплоскость.

Введем в $\mathbb{C}^{2}$ координаты $(z, w)$ так, что $M=\{\operatorname{Im} w=0\}$. Гиперповерхность $Y=\pi(M)$ делит поверхность Хопфа на две биголоморфно эвивалентные части $X_{ \pm}$, соответствующие $\mathbb{C}_{ \pm}^{2}=$ $\{ \pm \operatorname{Im} w>0\}$. Можно явно описать голоморфные функции на $X_{ \pm}$и убедиться, что области $X_{ \pm}$ штейновы. 
Рассмотрим для определенности область $X_{+}$. Голоморфной функции на $X_{+}$соответствует голоморфная функция на $\{(z, w) \mid \operatorname{Im} w>0\}$, инвариантная относительно умножения координат на $r$. Разложим такую функцию в ряд по $z$ с коэффициентами, голоморфными в верхней полуплоскости по переменной $w$ :

$$
f(z, w)=\sum_{n=0}^{\infty} f_{n}(w) z^{n}, \quad f_{n} \in \mathscr{O}(\{\operatorname{Im} w>0\}) .
$$

Условие инвариантности записывается в виде

$$
f_{n}(w)=r^{n} \cdot f_{n}(r w), \quad n \geqslant 0 .
$$

Пусть теперь голоморфиые в верхней полуплоскости $\{\operatorname{Im} w>0\}$ инвариантные функции $g_{1}, \ldots$, $g_{N}$ задают собственное голоморфное вложение штейнова многообразия $A$ в $\mathbb{C}^{N}$. Тогда отображение

$$
(z, w) \mapsto\left(g_{1}(w), \ldots, g_{N}(w), \frac{z}{w}\right) \in \mathbb{C}^{N+1}
$$

определяет собственное вложение $X_{+}$в $\mathbb{C}^{N+1}$.

Отметим одну особенность разрезания поверхности Хопфа. Возникающее на Леви-плоской гиперповерхности $Y$ слоение - это классическое слоение Риба на $S^{2} \times S^{1}$. Оно имеет единственный замкнутый слой - комплексный тор, соответствующий комплексной прямой $w=0$. Этот слой разделяет $S^{2} \times S^{1}$ на два полнотория. Все остальные слои суть комплексные прямые, "наматывающиеся" на этот тор. Полноторие, заполненное слоением такого вида, называется рибовской компонентой слоения.

Заметим, что на Леви-плоской гиперповерхности в кәлеровой комплексной поверхности слоение с рибовской компонентой возникнуть не может. Действительно, замкнутый слой слоения Риба гомологичен нулю, что невозможно для комплексной кривой в кэлеровой поверхности.

3. Определяющие функции. Цель этого пункта - показать, что построенные штейновы области с Леви-плоской границей существенно отличаются от строго псевдовыпуклых областей.

Пусть $U$ - область с гладкой границей $\partial U$ на гладком многообразии. Гладкая вещественнозначная функция $\psi$, определенная в некоторой окрестности $V \supset \partial U$, называется определяющей функцией для области $U$, если $U \cap V=\{x \in V \mid \psi(x)<0\}$ и, кроме того, $d \psi(x) \neq 0, x \in V$.

Хорошо известно, что если $U$ - строго псевдовыпуклая область на комплексном многообразии, то существует строго плюрисубгармоническая определяющая функция для $U$. Если же граница области не является строго псевдовьпуклой, то естественно рассмотреть плюрисубгармонические определяющие функции.

Оказывается, что для области с Леви-плоской границей множества уровня плюрисубгармонической определяющей функции никогда не являются строго псевдовыпукльми. Мы рассмотрим только случай $\operatorname{dim}_{\mathbb{C}} U=2$.

ПрЕДЛОЖЕнИЕ 2. Пусть $\psi$ - плюрисубгармоническая определяющая функиия области $U$ с гладжой Леви-плоской граничей на комплексной поверхности. Тогда гиперповерхности $\{\psi=t\}$ также Леви-плоские при малых $t \leqslant 0$.

ДоКАЗАТЕЛЬСтво. Пусть $U_{t}=\{\psi<t\}$. При малых $t \leqslant 0$ эта подобласть в $U$ имеет гладкую границу $\partial U_{t}$. Рассмотрим функцию $F=F(t),-\varepsilon<t \leqslant 0$, задаваемую формулой

$$
F(t)=\int_{\partial U_{t}} d^{c} \psi \wedge d d^{c} \psi
$$

Заметим, что в силу плюрисубгармоничности $\psi$ функция $F(t)$ неотрицательна.

Кроме того, из плюрисубгармоничности $\psi$ и формулы Стокса следует, что

$$
F(t)-F(s)=\int_{\partial U_{t}} d^{c} \psi \wedge d d^{c} \psi-\int_{\partial U_{s}} d^{c} \psi \wedge d d^{c} \psi=\int_{U_{t} \backslash U_{s}} d d^{c} \psi \wedge d d^{c} \psi \geqslant 0 \quad \text { при } t \geqslant s
$$

Таким образом, $F(t)$ - неубывающая функция при $-\varepsilon<t \leqslant 0$.

Остается заметить, что $F(t)=0$ тогда и только тогда, когда $\partial U_{t}-$ Леви-плоская гиперповерхность уровня функции $\psi$. 
СлЕдствиЕ. Двумерная штейнова область $U$ с компактной Леви-плоской әраницей не допускает плюрисуба армонических определяющих функиий.

ДоКАЗАТЕльство. Предположим, что существует плюрисубгармоническая определяющая функция области $U$. Тогда согласно предложению 2 найдется компактная Леви-плоская гиперповерхность $M \subset U$. Пусть $\varphi$ - строго плюрисубгармоническая функция в $U$. Ограничение $\left.\varphi\right|_{M}$ достигает максимума в некоторой точке $x \in M$. В частности, $\varphi$ достигает максимума на проходящем через эту точку листе слоения $M$ на комплексные кривые, что невозможно.

Эта заметка появилась в результате обсуждений затрагиваемых в ней вопросов с С. Оревковым, которому автор глубоко признателен за ценные советы и наблюдения.

\section{СПИСОК ЦИТИРОВАННОЙ ЛИТЕРАТУРЫ}

1. Немировский С. Ю. // Матем. заметки. 1998. Т. 63. № 4. С. 599-606. 2. Grauert H. // Math. Z. 1963. V. 81. P. 377-392. 3. Ohsawa T. // Publ. Res. Inst. Math. Sci. 1982. V. 18. P. 1185-1186.

Математический институт им. В. А. Стеклова РАН 